\title{
COMPARISON OF THEORETICAL AND OBSERVED INDICES FOR THREE PHOTOELECTRIC PHOTOMETRIC SYSTEMS
}

\author{
F. RUFENER and A. MAEDER \\ Geneva Observatory, Switzerland
}

\begin{abstract}
A comparison of the theoretical and observed indices has been made for the $U B V, u v b y$ and the Geneva's $U B V B_{1} B_{2} V_{1} G$ systems. In the actual state of the photometric calibrations, coincidence between theoretical and observed indices better than $0 \mathrm{~m} 1$ are probably not significant.
\end{abstract}

\section{Introduction}

The comparison of theoretical fluxes obtained by means of model atmospheres with observed fluxes can be made in at least two manners. By direct comparison with spectrophotometric recordings carefully calibrated for example relative to the black body. Another comparison is possible by means of a wide or an intermediate band photometry. One must then know the actual shapes of the pass-bands of the photometry considered. Since it is difficult to determine these directly with a few percent accuracy, the majority of the pass-bands of photometric systems have been calibrated by means of spectrophotometric recordings. The methods of calibration are numerous. Two difficulties complicate this indirect calibration, on the one hand the spectrophotometric reference generally ( $\alpha$ Lyr) posesses several absolute calibrations the respective qualities of which are not completely obvious; on the other hand, the instrument profile of the spectrophotometer is not always known explicitly and the recordings are published in the form of a limited number of fluxes measured in more or less 'clean' windows of the spectrum (the observed line blocking is often not mentioned).

This note intends to compare the observed sequences with those computed by means of theoretical model stellar atmospheres; this comparison is made with the three following photometries: Johnson-Morgan's $U B V$, Strömgrens $u v b y$ and Geneva's $U B V B_{1} B_{2} V_{1} G$. In each case, the same four models will be used, i.e. those by Mihalas (1966) describing main sequence stars between B8 V and A2 V. These are model atmospheres calculated with blanketing by hydrogen lines, but without metallic lines for $\log g=4$ and $\theta_{\text {eff }}=0.40 ; 0.45 ; 0.50 ; 0.55$. For each photometry, we use these models to compute the colour indices by following the procedures recommended by the authors who have calibrated the pass-bands of these systems. The computation program is due to E. Peytremann. The integrations are carried out by Simpson's method, except for the case of lines where the program automatically applies the trapezoidal method with an integration step that varies according to the region of the spectrum. The results obtained differ sometimes from those published by other authors. These differences must probably be attributed to different methods of integration. Here, the three comparisons are made by applying the same computational technique. 


\section{UB V Photometry}

The determination of this photometry's pass-bands has been the object of numerous comments which we will not repeat. A difficulty arises from the fact that the observations of the indices $U-B$ are not completely reduced to outside the atmosphere. On the other hand, the existent calibrations use different absolute spectrophotometric references. Let us consider three recent descriptions:

A. PROCEDURe Of AZUSIENIS AND STRAizys (1969)

In Table I of the paper by these authors, four response curves restore the probable profiles of the original natural system. These are the functions $\varphi_{U} p ; \varphi_{B} p ; \varphi_{B} ; \varphi_{V}$. We compute the natural indices by taking Mihalas (1966) models for $F_{\lambda}$

$$
\begin{aligned}
& C_{U-B}=2.5 \log \int F_{\lambda} \varphi_{B} p \mathrm{~d} \lambda-2.5 \log \int F_{\lambda} \varphi_{U} p \mathrm{~d} \lambda \\
& C_{B-V}=2.5 \log \int F_{\lambda} \varphi_{V} \mathrm{~d} \lambda-2.5 \log \int F_{\lambda} \varphi_{B} \mathrm{~d} \lambda
\end{aligned}
$$

The normalization proposed by the authors is

$$
\begin{aligned}
& U-B=C_{U-B}-1.33 \\
& B-V=C_{B-V}+0.67 .
\end{aligned}
$$

This has been established by means of spectrophotometric recording for which the calibration of $\alpha$ Lyr adopted by Code (1960) is considered.

\section{B. PROCEDURE OF MATTHEWS AND SANDAGE (1963)}

One must adopt for the three filters $U B V$ the response curves $S(\lambda)_{1}$ given in Table A1 by Matthews and Sandage (1963); these correspond to the natural response for the zenith of Mount-Wilson. The calculation of the natural indices of the models is obtained by means of the relations:

$$
\begin{aligned}
& (u-b)_{1}=2.5 \log \int F_{\lambda} S_{B}(\hat{\lambda})_{1} \mathrm{~d} \lambda-2.5 \log \int F_{\lambda} S_{U}(\hat{\lambda})_{1} \mathrm{~d} \lambda \\
& (b-v)_{1}=2.5 \log \int F_{\lambda} S_{V}(\lambda)_{1} \mathrm{~d} \lambda-2.5 \log \int F_{\lambda} S_{B}(\lambda)_{1} \mathrm{~d} \lambda .
\end{aligned}
$$

The transformations recommended by Matthews and Sandage (1963) to obtain the standard indices are

$$
\begin{aligned}
& U-B=0.921(u-b)_{1}-1.308 \\
& B-V=1.024(b-v)_{1}+0.81 .
\end{aligned}
$$

They have been obtained with spectrophotometric continua calibrated after the $\alpha$ Lyr description from Code (1960). No blocking correction is applied. 


\section{PROCEDURE OF MATSUSHIMA AND HALL (1969)}

That is the Matthews and Sandage's method modified only by the change of coefficients in the transformations to standard

$$
\begin{aligned}
& U-B=0.896(u-b)_{1}-1.288 \\
& B-V=0.982(b-v)_{1}+0.791 .
\end{aligned}
$$

These authors have computed them by filtering spectrophotometric recordings calibrated by Hayes' (1970) calibration of $\alpha$ Lyr. Moreover, they applied correction estimations for the effect of the hydrogen lines absent in the spectrophotometric continua (blocking correction).

Figure 1 represents with a dotted line the theoretical sequences A, B and C computed by means of the above mentioned procedures, for the four models of Mihalas (1966). The full line is the observed sequence defined by Johnson (1966).

\section{3. $u$ v b y Photometry}

According to Crawford (1966) the optical transmission of the filters is sufficient to define the pass-bands of the uvby photometry. It suffices to know in addition a normalisation constant for each filter (this is equal to saying that over the width of each filter one considers the response of the cell, the reflections of the mirror and atmospheric transmission to be free of chromatic effects). Matsushima (1969) has published optical profiles as well as the normalisation constants which calibrate the system. These result from a filtration of spectrophotometric continua coherent with the calibration of $\alpha$ Lyr by Hayes (1970). A particular correction is estimated to compensate for the effect of the $\mathrm{H} \delta$ line on the $v$ filter. No correction for blocking is necessary for the indices $u-b$ and $b-y$. We have applied this procedure to the four models by Mihalas. Figure 2 represents with a dotted line the resulting computed sequence. The observed sequence is the one established by Matsushima (1969) as being the best mean of observations for that region of the main sequence; this is shown with a full line.

\section{4. $U B V B_{1} B_{2} V_{1} G$ Photometry}

The pass-bands of the Geneva Observatory photometric system have been determined by Rufener and Maeder (1971). The method applies also spectrophotometric continua as means of calibration but is rather different from those used for the above mentioned photometries. Indeed, instead of establishing coherence between the spectrophotometric data and the actual observations by means of a linear transformation or by the choice of additive constants, this method seeks, by means of a representation by Fourier series, an optimum adjustment of the function $S(\lambda)=r(\lambda) s(\lambda) \varepsilon(\lambda)$ which, apart from giving the optical response of each filter also characterises the standard system. We have $r(\lambda)$ for the reflectivity of the telescope and the transmission of the Fabry lens. $s(\lambda)$ represents the electrical response of the photomultiplier. $\varepsilon(\lambda)$ corrects 


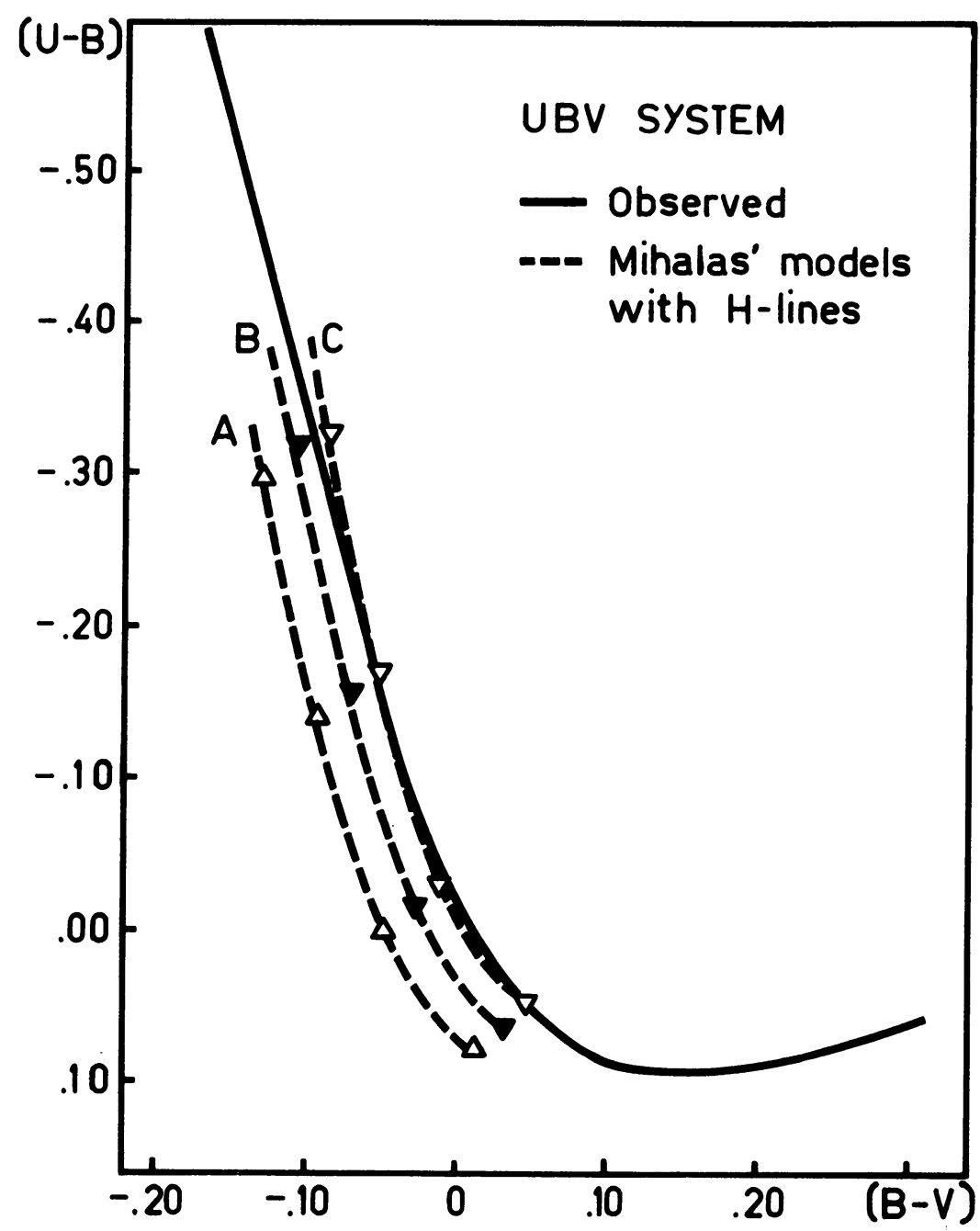

Fig. 1. Comparison of the observed and computed sequences for the response curves: (A) Azusienis and Straizys (1969); (B) Matthews and Sandage (1963); (C) Matsushima and Hall (1969).

the residual imperfections of the reductions outside the atmosphere. This function is close to unity.

Two calibrations of the reference star ( $\alpha$ Lyr) have been used, those by Code (1960) and by Hayes (1970). The spectrophotometric continua used were those of $O$ stars and subdwarfs showing very few strong lines. $55 \mathrm{Cyg}$ was also used but was weighted much less. No correction of blocking in the sense of Matsushima was made, Figure 3 shows Mihalas' models filtered with the Geneva photometry pass-bands; no normalisation is necessary. Dotted line A is according to Code's (1960) calibration, dotted line B according to Hayes (1970). The sequence with a full line is the observed sequence. 


\section{Conclusion}

For these three comparisons of the same models with the observed sequences, the fluctuation of the deviations from coincidence is essentially due to the errors of definition of the pass-bands. Various processes used by photometrists for observations and calibration of their system are much too inaccurate, their use should cease i.e.

(1) The partial reduction out atmosphere. That is to say ignoring the terms $\mathrm{K}_{2} \mathrm{C}$ and $K_{3}$ in the following expression

$$
m_{0}=m_{Z}-K_{1} F_{Z}-K_{2} C F_{Z}-K_{3} F_{Z}^{2},
$$

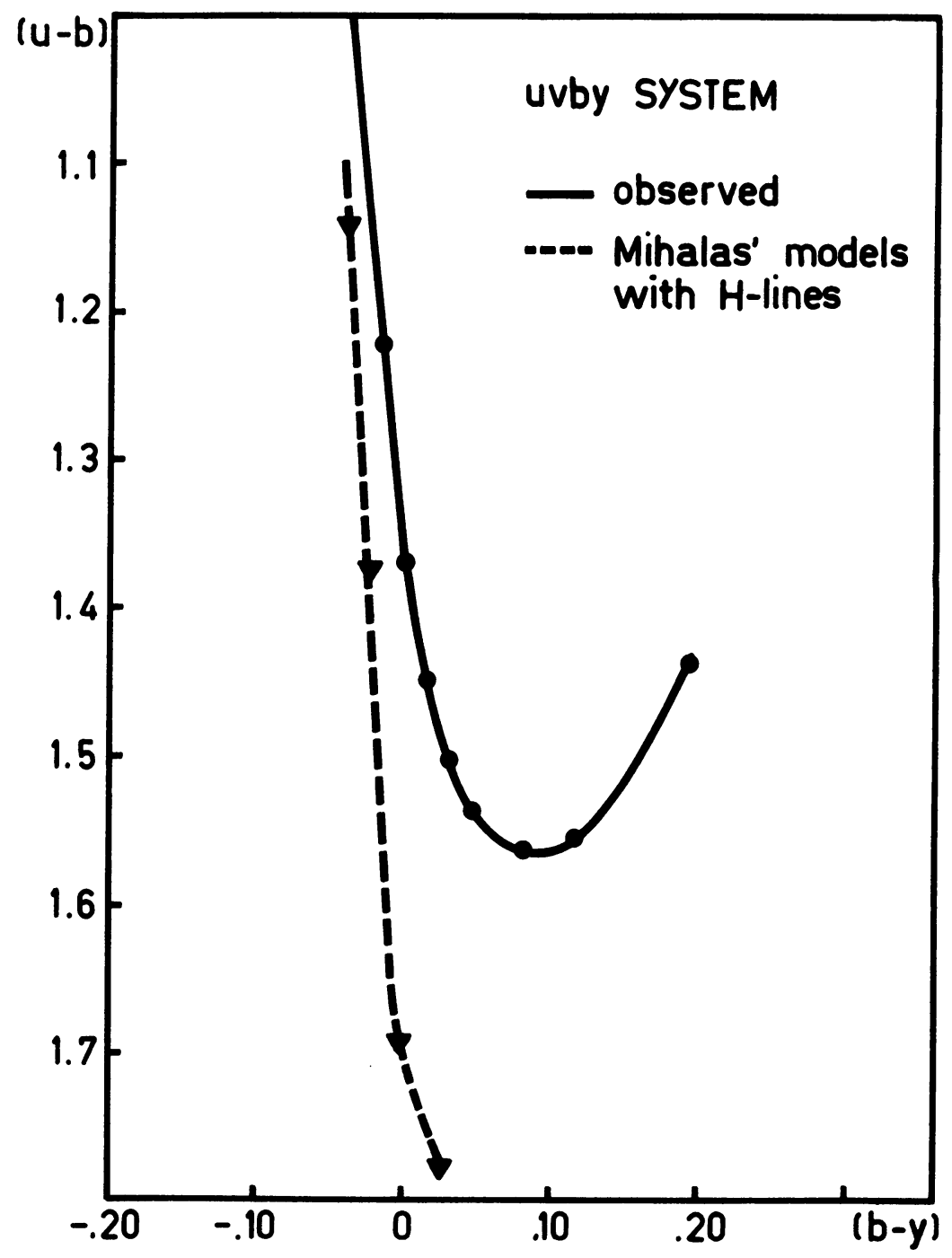

Fig. 2. Comparison of the observed and computed sequences following Matsushima (1969). 


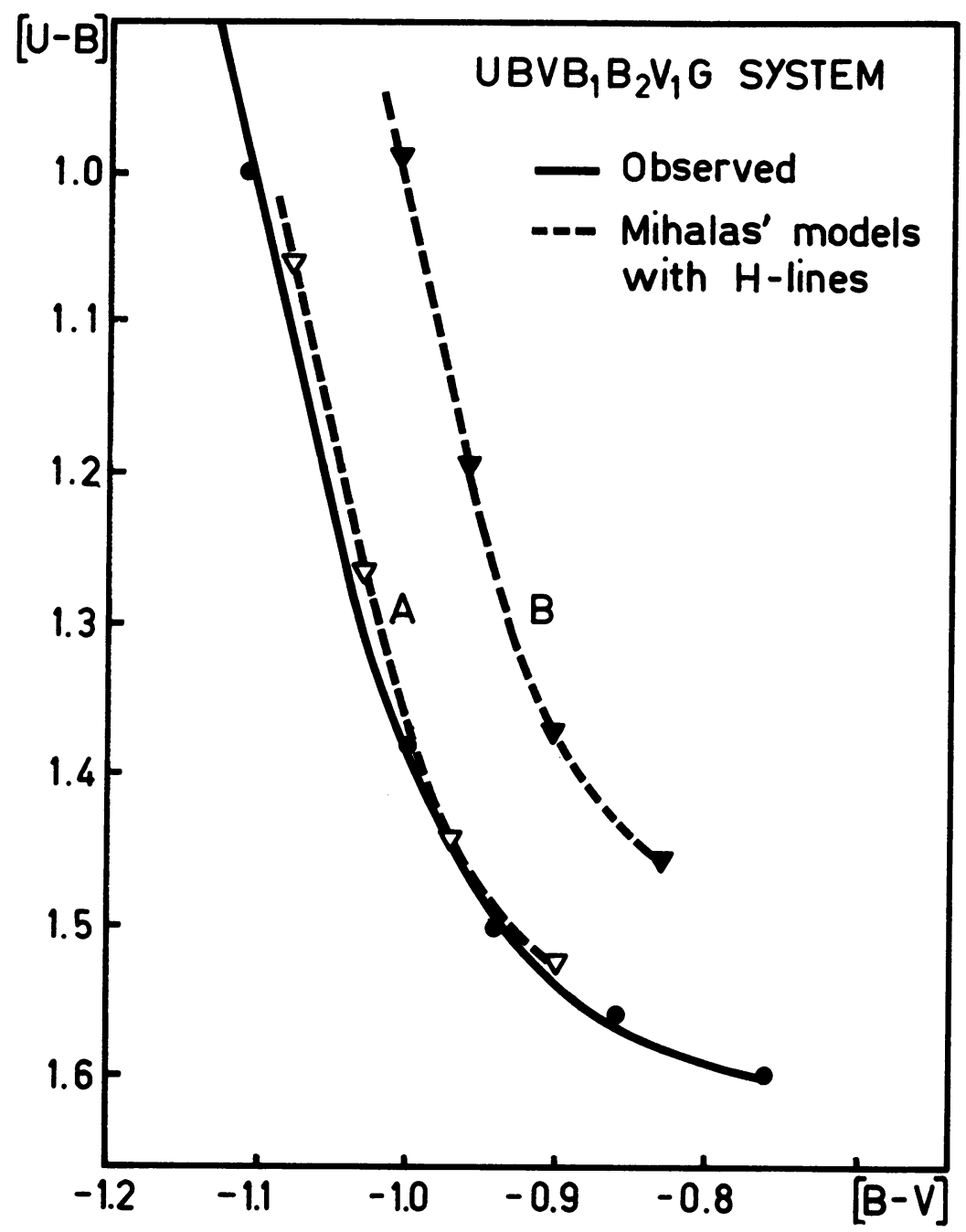

Fig. 3. Comparison of the observed and computed sequences with: (A) Code's calibration; (B) Hayes calibration.

where $m_{0}, m_{\mathrm{Z}}$ are the magnitudes respectively out atmosphere and at ground, $C$ is the colour index of the star, $F_{Z}$ the air mass and $K_{1}, K_{2}$ and $K_{3}$ the extinction coefficients.

(2) Identifying of the filter's optical response with response of the natural system. This implies, in the filter's spectral interval, to neglect the sensitivity variations of the $\mathbf{P M}$ as well as the mirrors reflecting power variations.

(3) The empirical estimate of blocking corrections applied to the calibrating spectrophotometric continua (frequently neglected correction).

(4) The fact to remedy to items 1 to 3 through a linear relation between the calculated and observed colours gives pass-bands only adapted to the stars considered for calibration (in general unreddened B and A stars). 
In the present state of the art concerning methods of calibration of these pass-bands, the coincidence of a theoretical sequence with an observed sequence is misleading and is certainly not more significant than a deviation from coincidence $<0^{\mathrm{m}}$. 1 . It is not easy to improve this situation. May be that a harmonisation of the methods of calibration and the use of continuous spectrophotometric descriptions calibrated properly for a selection of stars including in particular reddened $O$ stars would allow to reduce these uncertainties of calibration. An alternative solution would be the very careful global calibration of natural system (filter, PM, telescope) relatively to physical standards of radiation and the quasi simultaneous observations of the considered photometry standard stars.

\section{References}

Azusienis, A. and Straizys, V.: 1969, Soviet Astron. AJ 13 (2), 316.

Code, A. D.: 1960, Stars and Stellar Systems 6, 50.

Crawford, D. L.: 1966, in K. Lodén, L. O. Lodén, and U. Sinnerstad (eds.), 'Spectral Classification and Multicolour Photometry', IAU Symp. 24, 170.

Hayes, D. S.: 1970, Astrophys. J. 159, 165.

Johnson, H. L.: 1966, Ann. Rev. Astron. Astrophys. 5, 571.

Matsushima, S.: 1969, Astrophys. J. 158, 1137.

Matsushima, S. and Hall, D. L.: 1969, Astrophys. J. 156, 779.

Matthews, T. A. and Sandage, A. R.: 1963, Astrophys. J. 138, 30.

Mihalas, D.: 1966, Astrophys. J. Suppl. 13, 1.

Rufener, F. and Maeder, A.: 1971, Astron. Astrophys. Suppl. 4, 43.

\section{DISCUSSION}

Jaschek: I would just like to make a brief historical comment. At La Plata we have made a photometric catalogue (which is now at the printer) in which we have collected all photoelectric measurements made after those of Stebbins 1917. As a by-product we have tried to make out what color system each observer used. For the nearly two thousand references listed, we were uncapable of getting details for about one third, simply because the observers did not bother to tell if they used filters and what kind of photocells they used. Such a procedure is even today followed by some variable star observers who measure to $1 \%$ accuracy an undefined and irreproductible quantity. 